Research Article

\title{
A Parallel Strategy for High-speed Interpolation of CNC Using Data Space Constraint Method
}

\author{
Shuan-qiang Yang ${ }^{1,3}$, Jin-mei Wang ${ }^{2, *}$ and Zhui-liang Huang ${ }^{2}$ \\ ${ }^{1}$ College of Engineering, Fujiang Jiangxia University, Fuzhou 350108, China \\ ${ }^{2}$ College of All-Trans Logistics, Fuzhou University, Fuzhou 350108, China \\ ${ }^{3}$ School of Mechanical Engineering and Automation, Fuzhou University, Fuzhou 350108, China
}

Received 2 October 2013; Accepted 7 December 2013

\begin{abstract}
A high-speed interpolation scheme using parallel computing is proposed in this paper. The interpolation method is divided into two tasks, namely, the rough task executing in PC and the fine task in the I/O card. During the interpolation procedure, the double buffers are constructed to exchange the interpolation data between the two tasks. Then, the data space constraint method is adapted to ensure the reliable and continuous data communication between the two buffers. Therefore, the proposed scheme can be realized in the common distribution of the operation systems without real-time performance. The high-speed and high-precision motion control can be achieved as well. Finally, an experiment is conducted on the self-developed CNC platform, the test results are shown to verify the proposed method.
\end{abstract}

Keywords: CNC, Interpolation Scheme, Parallel Computing, Data Space Constraint

\section{Introduction}

With the development of PC software and hardware and network information technology, high-speed and highaccuracy $\mathrm{CNC}$ based on PC has become the research direction of modern $\mathrm{NC}$ technology. Its advantages owe to make use of the abundant resources of PC software and hardware, to achieve low cost and realize open architecture.

However, subjecting to the limitation of computer processing capacity, it results in the insufficiency of highspeed and real-time interpolation performance when used in dealing with large amounts of data transmission and highspeed machining. In the presented research strategies [3],[4],[5], a Real-Time Operating System (RTOS) is adapted to improve the real-time performance of the interpolation algorithm. The timing precision of the RTOS is generally at the range of several microseconds, which nearly satisfies the period timing requirements of high-speed realtime interpolation. Plenty of CPU time will be wasted during the interpolation task schedule, when high-speed motion control is being executed. It results in slowing CNC running speeds. On the other hand, the interpolation algorithms period cannot be too small, in order to guaranteed there is enough time to schedule the other system tasks. In addition, the current RTOS( is lack of unified program standard, thus, the software development is very difficult, and the code is inconvenient to port to the other platform. For example, Enhanced Machine Controller (EMC) project is presided by National Institute of Standards and Technology (NIST)[6].

* E-mail address: rucy03@163.com ISSN: $1791-2377$ C 2013 Kavala Institute of Technology. All rights reserved.
Therefore, it's necessary to develop a high-speed interpolation model for $\mathrm{CNC}$ system to meet the requirements of real-time machining and large data transmission.

2. Software and hardware function division of rough and fine interpolation and time sequence relationship between them

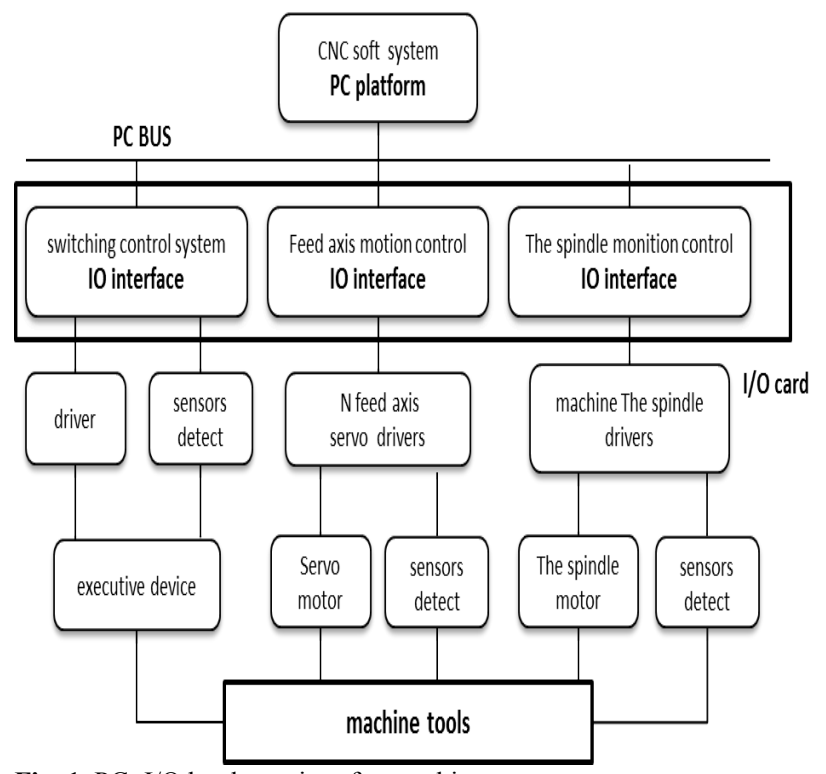

Fig. 1. $\mathrm{PC}+\mathrm{I} / \mathrm{O}$ hardware interface architecture 
As shown in Fig.1, a $\mathrm{PC}+\mathrm{I} / \mathrm{O}$ hardware interface architecture soft $\mathrm{CNC}$ system has been developed. For the high-speed machining and the large NC code transmission, it's difficult to achieve prospective control effect with single PC, to real-time periodically implement the rough and fine interpolation task. In fact, in the process of rough and fine interpolation, the fine interpolation task real-time executes strictly according to sample period of rough interpolation. As to rough interpolation computing, just provide the increment data of the trajectory to fine interpolation task in time. Therefore, the increment data of the trajectory transmission between them is not necessary to execute in real-time.

In this software and hardware combination platform, an interpolation algorithm though time-slicing method is adapted to split the tool trajectory and control the movement of the machine tool. The rough and fine interpolation tasks are independent with each other, but the time sequence between them should be kept in order. The main task of rough interpolation accomplished by PC is to calculate the increment data of each axis within single interpolation period, and then the data will be changed into pulses number to the fine interpolation task. Accordingly, the fine interpolation task running in $\mathrm{I} / \mathrm{O}$ card evenly distributed the pulse singles within the interpolation period to the servo drivers. The fine interpolation algorithm is relative simple, the output frequency is high, and a good real-time performance is needed.

\section{The parallel strategy for high-speed interpolation based on data space constraint}

Fig.2 shows that the proposed parallel interpolation model based on data space constraints. It is designed to ensure the reliability and continuity of high-speed data transmission between the rough and fine interpolation tasks. The double buffer is created to change the time sequence into the data space constraint. That is, two FIFO (first in first out) buffer are set up in the PC and the I/O interface card respectively.

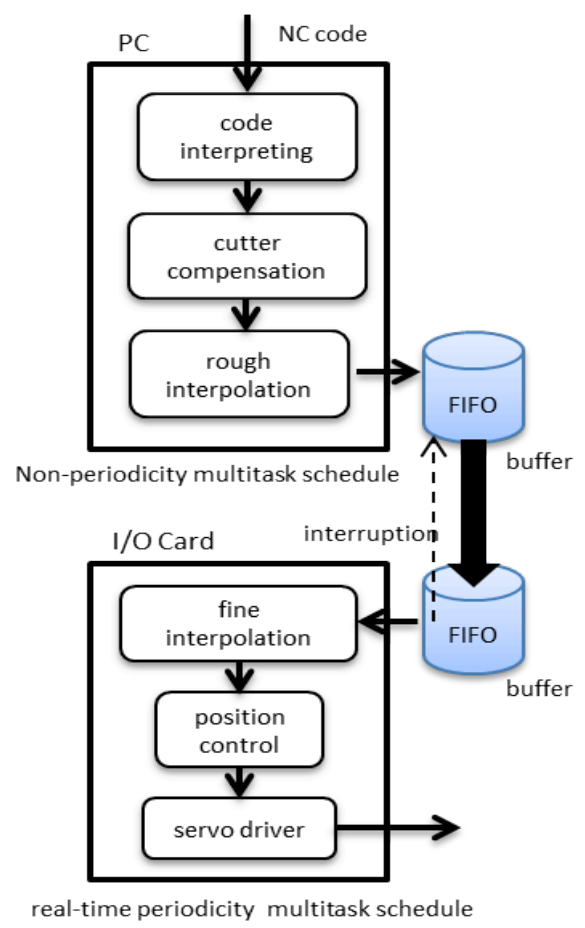

Fig. 2 Rough and fine parallel high-speed interpolation model based on data constraint
Then the parallel calculation is substituted to the serial calculation to improve the efficient of the real-time interpolation. In this strategy, the fine interpolation task executes precisely, periodically and strictly with the sample period of the rough interpolation task. Meanwhile, the rough interpolation task takes possession of more free calculation time to implement continuous interpolation operation. The key issue of the posed method is data space constraint between the rough and fine interpolation tasks. The buffer in the I/O card cannot be empty before the end of the trajectory interpolation. The rough interpolation task produces the increment data from the tool trajectory, and the fine one consumes the data to generate the pulse signals. Hence, they can be seen as the producer-consumer model (Fig.3).

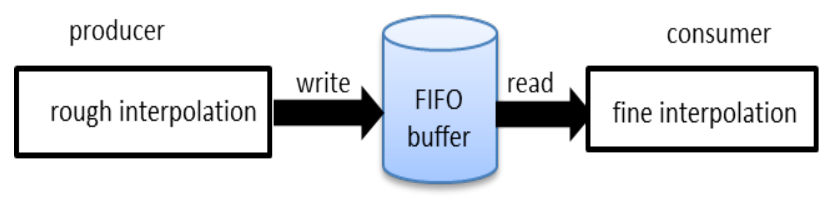

Fig.3 Producer-consumer model

In this model, the fine interpolation is a timing period task and the consuming velocity of the data in the buffer is constant. If the average velocity of the data produced from the rough interpolation task is less than the consuming of the fine interpolation, the machining process will be suspended for the insufficiency of the producing data. Therefore, the average time of a new increment data calculated by the rough interpolation task, should be less than the preset interpolation period. It the only way to satisfy the data space constrain between the rough and fine interpolation tasks, and to ensure the real-time of the whole motion control system.

The Real-time rough interpolation calculation is cancelled in this interpolation strategy that avoids wasting the large amounts of CPU time on real-time interruption schedule. As long as data space constraint between the rough and fine interpolation are satisfied, the real-time tasks schedule capacity of the PC is not an essential condition as the presented methods. It creates the conditions for highspeed interpolation and adapting complicated interpolation algorithm. Furthermore, the system software of CNC can be built on a standard distribution without requiring real-time capacity. It can make full use of the kinds of software resources and standard development tools on PC platform, and realize more conveniently for the openness of system.

\subsection{Double buffer synchronous mechanism}

The fatal error will appears in two situations when highspeed machining is executing. One is the FIFO buffer in the $\mathrm{I} / \mathrm{O}$ card is exhausted by the fine interpolation task before machining finish, the other is the buffer is overflow because too many data are received from the rough interpolation task. Thus, a double buffer synchronous mechanism is proposed in this paper to ensure the reliable data transmission between the rough and fine interpolation tasks. When the data commination is started between the PC and $\mathrm{I} / \mathrm{O}$ card, the interrupt mechanism is applied when the space of the buffer in the $\mathrm{I} / \mathrm{O}$ card is less than the preset value. New interpolation data will be sent to the I/O card from the PC when the interrupt is tricked and handled. Choosing a proper constant length of the data package from PC, and the preset data space in the $\mathrm{I} / \mathrm{O}$ card are becoming a key issue in the proposed method. 


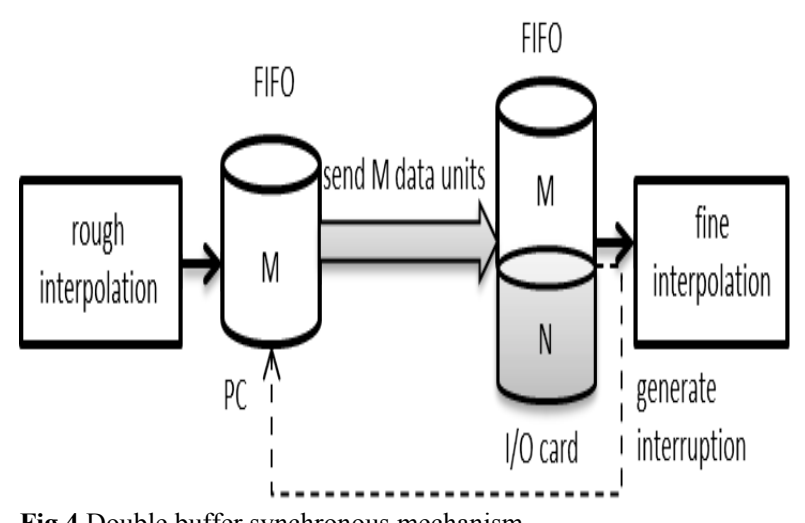

Fig.4 Double buffer synchronous mechanism

The process of the synchronous mechanism by this method is shown in Fig.4. If the data units of the FIFO buffer in the $\mathrm{I} / \mathrm{O}$ card is $(\mathrm{M}+\mathrm{N})$, where $\mathrm{N}$ is the threshold value tricking the interrupt in $\mathrm{I} / \mathrm{O}$ card and $\mathrm{M}$ is the constant numbers of the interpolation data from the PC.

When the data space left in the buffer of the $\mathrm{I} / \mathrm{O}$ card is equals to $\mathrm{N}$, an interrupt will be tricked. Once the PC responses to the interrupt, a constant number $\mathrm{M}$ of the data produced by the rough interpolation task is sent to $\mathrm{I} / \mathrm{O}$ card from the buffer in the PC. The total time of the single data transmission time, and the response time in the PC when the interrupt is handled can be seen as approximately the same. It can be represented by the variable $t$. If the interpolation period is set as $T$, the number of the data units left in the buffer in the $\mathrm{I} / \mathrm{O}$ card is $(\mathrm{N}-\mathrm{t} / \mathrm{T})$ until a new interpolation data is received. To guarantee the buffer in the $\mathrm{I} / \mathrm{O}$ card cannot be exhausted by the fine interpolation task, the condition $(\mathrm{N}>\mathrm{t} / \mathrm{T})$ must be satisfied. The newly received data number cannot exceed the unused data space, to avoid the situation that the buffer in the card is overflow. Then the condition $(\mathrm{M}+\mathrm{N}-\mathrm{t} / \mathrm{T}<\mathrm{M}+\mathrm{N})$ should be met as well. In addition, if there are some free spaces in the interpolation data buffer in the PC, the rough task is aroused to run again. Newly calculated data is added into the buffer until the buffer is full. Then the rough interpolation task is suspended and turned into sleep status.

\subsection{Analysis of selecting the size of data buffer $M, N$}

According to synchronous work mechanism, fig. 3 shows relative time sequence when the rough and fine interpolation tasks run simultaneously. The relationship between them are expressed as follow

$$
\left\{\begin{array}{l}
t_{n}<N T \\
t_{d}+t_{c}<t_{m} \\
t_{m}=M T
\end{array}\right.
$$

$t_{n}$ : the total time of $\mathrm{PC}$ interrupt response and data transmission

$t_{m}$ : the running time from the number of data units in $\mathrm{I} / \mathrm{O}$ card from $\mathrm{M}+\mathrm{N}$ to $\mathrm{N}$

$t_{d}$ : the schedule time of awakening the rough interpolation task

$t_{c}:$ the time from the rough interpolation task start and full fills the FIFO buffer

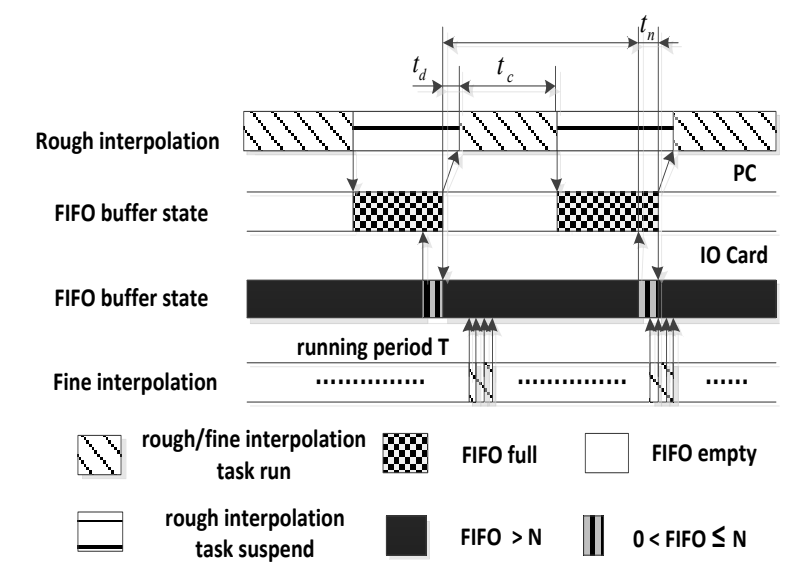

Fig.5 Rough/fine interpolation and buffer state time sequence relationship

As shown in Fig.5, if the value of $\mathrm{N}$ is too small, the FIFO buffer in the I/O card will be empty before machining finish. The machining procedure will be terminated caused by this fatal error. In contrast, a large number of $\mathrm{N}$ will result in the waste of the data space in the buffer. Selecting a proper value of $\mathrm{M}$, is not only to ensure the high-speed interpolation computing, but also to guarantee there have enough time to schedule other tasks in the system. There is a key point should be considered. As the preset value of $M$ is greater, the value of $t_{m}$ is longer. The movement of the machine will be delayed for a longer time after the interpolation task started. Therefore, the proper value of $M$ and $\mathrm{N}$ are set as the proposed method is very important.

\section{Experiment}

To verify the effectiveness of the proposed method, a serial of experiments has been designed on the self-developed CNC platform. As shown in Fig.6, a standard distribution of Linux 2.6 is selected as the software system, and the I/O card based on FPGA chips is applied to control the servo motors of the CNC machine.

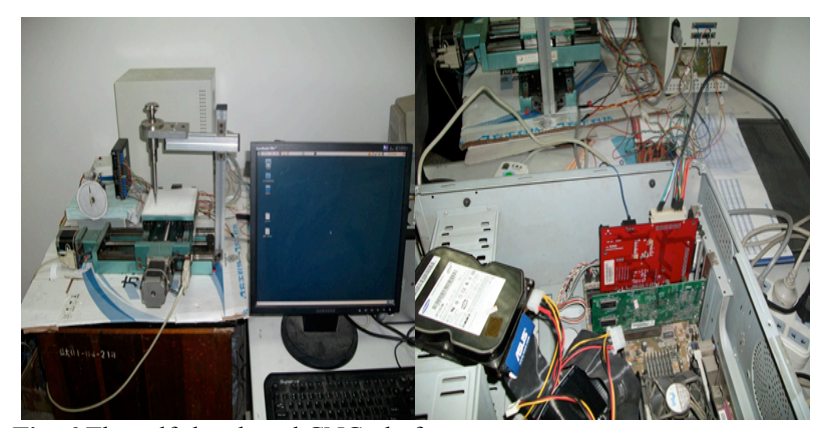

Fig. 6 The self-developed CNC platform

4.1Rough interpolation computing time test in PC soft In the proposed parallel strategy, the rough interpolation task is used to sample the machining trajectory. And the average time $t$ of a new increment data calculated by the rough interpolation task, should be less than the preset interpolation sampling period $\mathrm{T}$. When conducting the highspeed interpolation computing, the sampling period $\mathrm{T}$ is general set at millisecond level. Therefore, it's necessary to test the average rough interpolation computing time in PC soft under different sampling period $\mathrm{T}$ at millisecond level. 


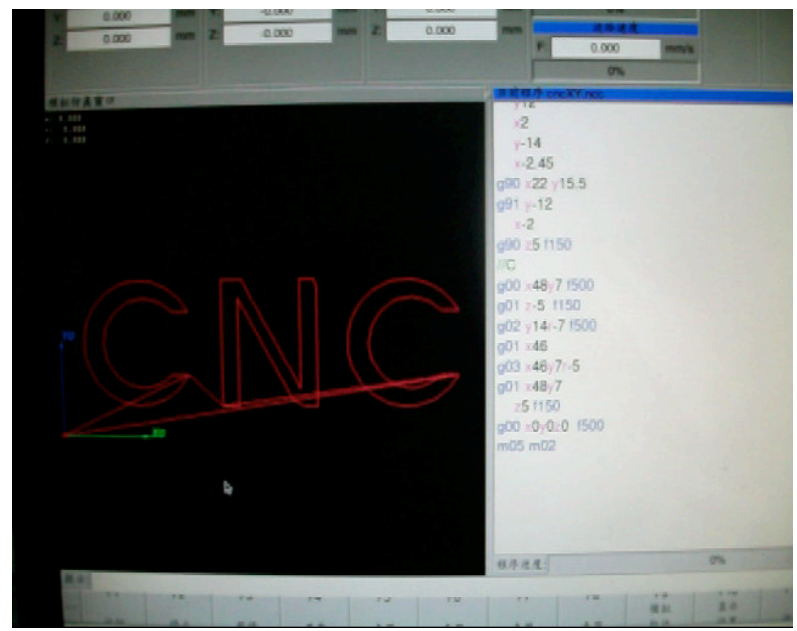

Fig. 7 The characters of CNC with continuous short-segments

As shown in Fig. 6, a program machining the characters of $\mathrm{CNC}$ with continuous short-segments is used to test the proposed interpolation scheme. In this test, PC soft schedule other tasks normally regardless of fine interpolation task in $\mathrm{I} / \mathrm{O}$ card, namely, the FIFO buffer in PC will be read as long as the FIFO is full.

The interpolation period is set as: $4 \mathrm{~ms}, 2 \mathrm{~ms}, 1 \mathrm{~ms}$ and $0.1 \mathrm{~ms}$. The results are shown in the Table 1:

Table 1 The testing results of rough interpolation computing

\begin{tabular}{c|c|c|c}
\hline $\begin{array}{c}\text { Interpolation } \\
\text { sampling } \\
\text { period } \\
(\mathrm{ms})\end{array}$ & $\begin{array}{c}\text { The number } \\
\text { of } \\
\text { Interpolation } \\
\text { sampling } \\
\text { point }\end{array}$ & $\begin{array}{c}\text { The total } \\
\text { time of } \\
\text { interpolation } \\
\text { computing } \\
\text { (us) }\end{array}$ & $\begin{array}{c}\text { The average } \\
\text { time of every } \\
\text { Interpolation } \\
\text { sampling point } \\
\text { computing(us) }\end{array}$ \\
\hline 4 & 43760 & 543846 & 12.428 \\
\hline 2 & 119023 & 636128 & 5.345 \\
\hline 1 & 202920 & 746129 & 3.677 \\
\hline 0.1 & 3035454 & 3291068 & 1.084 \\
\hline
\end{tabular}

We can see from the table, the smaller of Interpolation sampling period $\mathrm{T}$, the larger of the number of Interpolation sampling point. And the total time of interpolation computing is longer as well. But the average time $t$ of every Interpolation sampling point computing is all less than preset sampling period $\mathrm{T}$.

\subsection{Interpolation data transmission test using the double buffer synchronous mechanism}

Table 2 The test scenarios of the interpolation data transmission

\begin{tabular}{c|c|c|c}
\hline $\mathrm{M}$ & $\mathrm{N}$ & $\mathrm{T}(\mathrm{ms})$ & Error \\
\hline 1 & 1 & $4,2,1,0.1$ & No \\
\hline 2047 & 1 & $4,2,1,0.1$ & No \\
\hline
\end{tabular}

To verify the effectiveness of the proposed double buffer synchronous mechanism, a data Interpolation transmission testing is conducted. The test scenarios of the interpolation data transmission are shown in the Table 2.M is the constant numbers of the interpolation data transmission, $\mathrm{N}$ is the threshold value tricking the interrupt, and $\mathrm{T}$ is fine interpolation running period. There are two kinds of extreme cases which are considered about $\mathrm{M}$ and N. For each case, when change the value of $\mathrm{T}$ from $4 \mathrm{~ms}$ to $0.1 \mathrm{~ms}$, watch to see whether error occurred during the data transmission process. After repeated testing, there are no error occurred. The validity and feasibility of the method are verified.

\subsection{A machining example}

As shown in Fig. 7, a program machining the characters of $\mathrm{CNC}$ with a serial of line segments is used to demonstrate the proposed interpolation scheme. In the experiment, the interpolation period is set as $2 \mathrm{~ms}$, the volume setting of FIFO in I/O interface card is 2048 data units (a data units is $32 \mathrm{bit}$ ) and $\mathrm{N}$ is 48 data units. The length of transmission data $\mathrm{M}$ is 2000 data units as well.

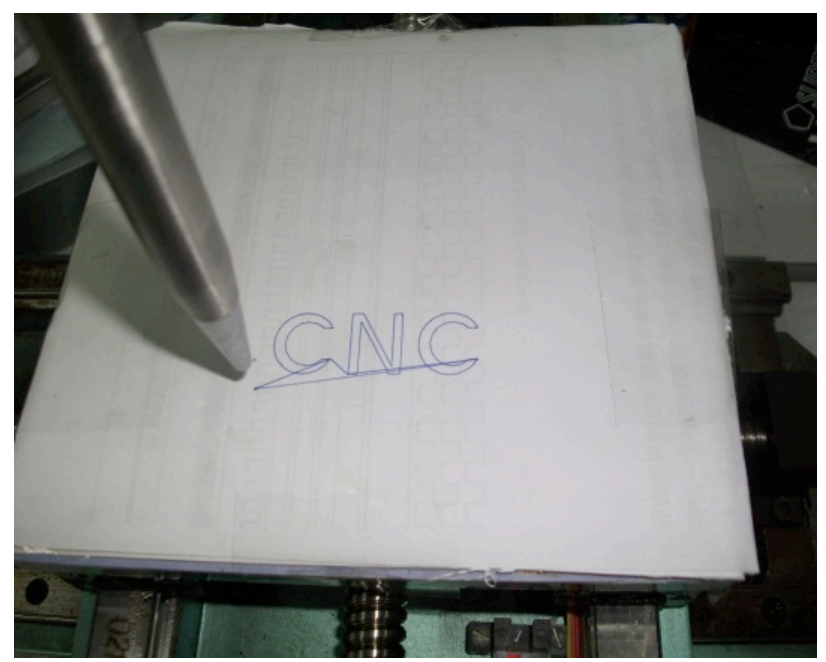

Fig. 8 A machining sample

The practical test results show the CNC system based upon the proposed method has high effective performance, and realizes real-time and high-speed interpolation computing.

\section{Conclusions}

In this paper, a parallel strategy is applied to the high-speed interpolation computing. The rough interpolation task is used to sample the machining trajectory, and the fine one to generate pulses to the servo drivers. They are running simultaneously to realize real-time interpolation based upon the data space constraint. In addition, a synchronism method is developed for the double buffer in the PC and I/O card. The rough task is running continuous without real-time periodically scheduled. Then, a high-speed interpolation computing can be obtained, and a high performance of CNC system can be achieved. Finally, the effectiveness of the proposed method is verified by a practical experiment.

\section{Acknowledgements}

The author would like to acknowledge the support of the special subject of fujian province higher educational science and technology educational fund (JK2012057).

\section{References}

1. XU Ning, YANG Xiuyun. The current situation and development of the computer numerical control system. Machinery Design and Manufacture, 2006, (4): 132-134.
2. WANG Chengyuan, CHANG Guoxiang, Guo Yumei. Development state and tendency of Open NC system. Journal of Shenyang University of Technology, 2007, 29(1): 61-64. 
Shuan-qiang Yang, Jin-mei Wang and Zhui-liang Huang

IJournal of Engineering Science and Technology Review 6 (3) (2013) 41 - 45

3. CHEN Zongyu, GUO Wei, et al. Research on open CNC system based on Windows NT and RTX. Computer Integrated Manufacturing Systems, 2006, 12(4): 568-572,640.

4. LIANG Hongbin, WANG Yongzhang. Real-time performance of a Windows-based open architecture $\mathrm{CNC}$ system. Computer Integrated Manufacturing Systems. 2003, 4(9):403-406.

5. ZHU Dayu, LI Yan, et al. RTLinux-based software CNC system. Computer Integrated Manufacturing Systems, 2004, 12(10):15711576.

6. SHACKLEFORD W P, PROCTOR F M. Use of open source distribution for a machine tool controller [EB/OL].[2008-05-24]. http://www.isd.mel.nist.gov/documents/shackleford /4191_05.pdf 